\title{
OCORRÊNCIA DE Cyrtoplax spinidentata (BENEDICT, 1892) (CRUSTACEA : BRACHYURA: GONEPLACIDAE) PARA O LITORAL DO ESTADO DO CEARÁ, BRASIL
}

\author{
Luis Ernesto Arruda BEZERRA ${ }^{1 *}$ \\ Cristina de Almeida ROCHA-BARREIRA ${ }^{2}$ \\ Aline Ferreira da SILVA ${ }^{3}$ \\ ${ }^{1}$ Universidade Federal do Ceará, Centro de Ciências, Departamento de \\ Biologia. Campus do Pici, Av. Mister Hull, s/n. 60455-760 Fortaleza, \\ CE (luis_ernesto2@yahoo.com.br). \\ ${ }^{2}$ Universidade Federal do Ceará, Instituto de Ciências do Mar \\ (LABOMAR). Av. da Abolição, 3207. 60165-081 Fortaleza, CE \\ (cristina@labomar.ufc.br). \\ ${ }^{3}$ Mestranda do Programa de Pós-Graduação em Ciências Marinhas \\ Tropicais do Instituto de Ciências do Mar (LABOMAR), Universidade \\ Federal do Ceará. (alinefbio@yahoo.com.br). \\ *Autor correspondente.
}

Recebido: $10 / 05 / 05$

Aceito: $27 / 06 / 05$

\section{RESUMO}

O gênero Cyrtoplax é representado no litoral brasileiro por apenas uma espécie, $C$. spinidentata, a qual tem ocorrência conhecida para os Estados de Pernambuco, Alagoas, Bahia e Rio Grande do Sul. Além do litoral brasileiro, essa espécie pode ser encontrada nas Antilhas e no Uruguai. Um exemplar de C. spinidentata foi coletado no estuário do Rio Pacoti, município de Aquiraz, Ceará $\left(03^{\circ} 43^{\prime} \mathrm{S}\right.$; $\left.38^{\circ} 32^{\prime} \mathrm{W}\right)$, em substrato de lama, temperatura de $32^{\circ} \mathrm{C}$, salinidade 36 e $1,23 \mathrm{mg} / \mathrm{L}$ de oxigênio dissolvido, sendo este o primeiro registro dessa espécie para o litoral do Estado do Ceará, ampliando assim a distribuição setentrional dessa espécie na costa brasileira.

Palavras-chave: Cyrtoplax spinidentata, primeiro registro, Estado do Ceará.

\section{ABSTRACT \\ OCCCURENCE OF Cyrtoplax spinidentata (BENEDICT, 1892) (CRUSTACEA: BRACHYURA: GONEPLACIDAE) IN THE COAST OF CEARÁ STATE, BRAZIL}

The genus Cyrtoplax (Benedict, 1892) is represented in Brazil by only one species $C$. spinidentata. This species has been referred in Brazil, until now, for the states of Pernambuco, Alagoas, Bahia, São Paulo e Rio Grande do Sul. This work reports, for the first time, C. spinidentata to the Coast of Ceará State. The present record extends the geographic range known for this species in the Brazilian coast.

Key-words: Cyrtoplax spinidentata, Ceará sate, first record.

Tropical Oceanography, Recife, v. 33, n. 2, p. 119-122, 2005. 


\section{INTRODUÇÃO}

Cyrtoplax spinidentata (Benedict, 1892) é uma espécie que vive em substratos inconsolidados, ocorrendo desde águas rasas, até aproximadamente $150 \mathrm{~m}$ (MELO, 1996). Sua ocorrência na zona costeira parece ser rara, uma vez que poucos exemplares foram coletados nessa região, sendo mais comuns na plataforma continental, onde pode ser encontrado junto com outras espécies de Brachyura como Libinia spinosa H. Milne Edwards, 1834, Leurocyclus tuberculosus (H. Milne Edwards; Lucas, 1843), Portunus spinicarpus (Stimpson, 1871) e Chasmocarcinus rathbuni Bouvier, 1917 (BORDIN, 1987).

Segundo Coelho et al., (2004), esse caranguejo escava tocas em áreas de sedimento fluido, onde pode ser encontrada uma fauna associada característica, a qual inclui três espécies de camarões da família Alpheidae (Alpheus sp., Salmoneus ortmanni (Rankin, 1898) e Leptalpheus petronii Ramos-Porto; Souza, 1994), o Stomatopoda Cloridopsis dubia (Milne-Edwards, 1837) e os peixes Gobionellus smaragdus, (Valenciennes, 1837), G. boleosoma (Jordan; Gilbert, 1882) e Erotelis smaragdus (Valenciennes, 1837).

Essa espécie é geralmente encontrada em áreas de salinidade alta (BORDIN, 1987); entretanto, registros de $C$. spinidentata para áreas estuarinas foram feitos por Coelho; Ramos (1972) e Coelho-Santos (2001). O presente trabalho reporta pela primeira vez a ocorrência de um exemplar de C. spinidentata para o litoral do Estado do Ceará, coletado no estuário do Rio Pacoti, sendo este o registro mais ao norte dessa espécie no litoral brasileiro.

\section{MATERIAIS E MÉTODOS}

O material estudado foi coletado no estuário do Rio Pacoti, município de Aquiraz, Ceará $\left(03^{\circ}\right.$ 43’ S; $38^{\circ} 32^{\prime} \mathrm{W}$ ), em um banco de lama que fica exposto apenas na maré baixa. A salinidade e temperatura, assim como oxigênio dissolvido do local, foram mensurados no momento da coleta com o auxilio de um refratômetro, termômetro e oxímetro, respectivamente, e são fornecidos no item dados ecológicos.

Foi realizada ainda a análise sedimentológica do substrato onde o exemplar foi coletado, a qual baseou-se na determinação do percentual de umidade, teor de matéria orgânica e análise granulométrica, seguindo-se o método proposto por Jeffery et al., (1992) para matéria orgânica e Suguio (1973) para análise granulométrica.

Após a coleta, os exemplares foram fixados em etanol 70\% e identificados. Os animais serão depositados na Coleção Carcinológica do Instituto de Ciências do Mar (LABOMAR) da Universidade Federal do Ceará, Fortaleza. No item material examinado são utilizadas as seguintes abreviaturas: $\mathrm{CC}=$ comprimento da carapaça e LC = largura da carapaça. A classificação taxonômica adotada segue a proposta por Martin; Davis (2001).

\section{RESULTADOS}

Infraordem Brachyura Latreille, 1803

Seção Eubrachyura de Saint Laurent, 1980

Superfamília Xanthoidea MacLeay, 1838

Família Goneplacidae MacLeay, 1838

Gênero Cyrtoplax Rathbun, 1914

Cyrtoplax spinidentata (Benedict, 1992)

Material examinado: 1 macho (CC X LC = 13,2 X 9,3 mm), 10.II.2005, estuário do Rio Pacoti,

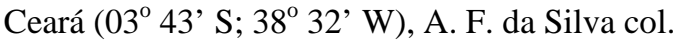


Distribuição geográfica: Atlântico Ocidental - Antilhas, Brasil: Pernambuco (COELHO; SANTOS, 1989), Alagoas (RATHBUN, 1918), Bahia (GOUVÊA, 1971; COELHO; RAMOS, 1972), São Paulo (BERTINI; FRANSOZO; MELO, 2004); Rio Grande do Sul (BORDIN, 1987) e Uruguai (BORDIN, op cit.).

Habitat: Em fundos de lama, eventualmente em areia. Águas rasas até 150 m (MELO, 1996).

Dados ecológicos: Exemplar coletado com amostrador do tipo "Core" a aproximadamente $10 \mathrm{~cm}$ de profundidade, em salinidade de 36 , temperatura de $32^{\circ} \mathrm{C}$ e com $1,23 \mathrm{mg} / \mathrm{L}$ de oxigênio dissolvido. Houve uma predominância de areia fina e argila na área coletada, tratando-se assim de uma região com predominância de sedimentos finos. O percentual de matéria orgânica foi de 3,18 \pm 0,16 \% e a umidade foi de $22,9 \%$

\section{COMENTÁRIOS}

O caranguejo Cyrtoplax spinidentata é um animal típico da região do infralitoral, e parece ser raro nas águas do litoral do Nordeste brasileiro, sendo mais comum na região Sul do país. CoelhoSantos (2001) realizou um extenso levantamento da fauna de crustáceos decápodes que ocorrem no estuário do Rio Paripe, Pernambuco e registrou apenas 3 exemplares dessa espécie. Já Bordin (1987) assinalou a presença de 48 exemplares coletados em uma única campanha oceanográfica na plataforma continental do Estado do Rio Grande do Sul e áreas adjacentes, sendo predominantes no inverno e em fundos de lodo e argila.

Coelho-Santos (2001) registrou essa espécie em áreas onde predominou no sedimento a fração areia fina, com o grau de umectação entre $29,13 \%$ e 50,01\%, a temperatura entre 29,1 e $34,1^{\circ} \mathrm{C}$ e salinidade entre 30,54 e 36,02. Da mesma forma Bordin (op cit.) registrou essa espécie para áreas de sedimento fino, com salinidade entre 32,8 e 36,1 temperatura entre 9,5 e $22,2{ }^{\circ} \mathrm{C}$ e oxigênio dissolvido entre 3,21 e 6,51 mg/L.

A granulometria do local de coleta no estuário do Rio Pacoti, demonstra a preferência de $C$. spinidentata por sedimentos finos, estando assim de acordo com o que diz a literatura sobre o habitat dessa espécie. A salinidade do local, em torno de 36, confirma a ocorrência desse braquiúro em áreas de salinidade alta.

\section{AGRADECIMENTOS}

Os autores agradecem ao Prof. M.Sc Alexandre Oliveira de Almeida da UESC/BA pela revisão do manuscrito, ao biólogo do LABOMAR/UFC M.Sc Wilson Franklin Júnior, a bióloga Rossana Cristina de Azevedo Souza e a graduanda Adriana de Freitas Diniz pela ajuda no campo e na coleta do material.

\section{REFERÊNCIAS BIBLIOGRÁFICAS}

BERTINI, G.; FRANSOZO, A.; MELO, G.A.S. Biodiversity of brachyuran crabs (Crustacea: Decapoda) from non-consolidated sublittoral bottom on the northern coast of São Paulo State, Brazil. Biodiversity and Conservation, Amsterdan, v.13, n.12, p. 2185-2207, 2004.

BORDIN, G. Brachyura da plataforma continental do Estado do Rio Grande do Sul, Brasil e áreas adjacentes (Crustacea, Decapoda). Iheringia, Série Zoologia, Porto Alegre, v. 66, p. 3-32, 1987. 
COELHO, P.A.; RAMOS, M de A. A constituição e a distribuição da fauna de crustáceos do litoral leste da América do Sul entre as latitudes $5^{\circ} \mathrm{N}$ e $39^{\circ} \mathrm{S}$. Trabalhos Oceanográficos da Universidade Federal de Pernambuco, Recife, v. 13, p. 133-236, 1972.

COELHO, P.A.; SANTOS, M.A.C. Crustáceos Decápodos e Estomatópodos do Rio Paripe, Itamaracá - Pe. In: Trabalhos do VII Encontro de Zoologia do Nordeste. 1989, Maceió, Anais da Sociedade Nordestina de Zoologia, Maceió, 1989, p. 43-62.

COELHO-SANTOS, M.A. Crustáceos decápodos do mediolitoral do estuário do Rio Paripe Itamaracá, Pernambuco, Brasil. Recife, 2001. 137p. Tese (Doutorado em Oceanografia) Departamento de Oceanografia. Universidade Federal de Pernambuco.

GOUVÊA, E.P. Ocorrência de Cyrtoplax spinidentata (Crustacea: Decapoda: Goneplacida) no litoral brasileiro. Ciência e Cultura, São Paulo, v. 23 (Supl.), p.391. 1971.

JEFFERY, G.H.; BASSETT, J.; MERIDHAM, J.; DENEY, R.C. Vogel - Análise química quantitativa. Rio de Janeiro, Guanabra Koogan, 1992. 351p.

MARTIN, J.W.; DAVIS, G.E..An updated classification of the recent Crustacea. Natural History Museum of Los Angeles County, Science Series, Los Angeles, v. 39, p. 1-124, 2001.

MELO, G.A.S. Manual de identificação dos Brachyura (Caranguejos e siris) do litoral brasileiro. São Paulo, Ed. Plêiade, 1996. 604p.

RATHBUN, M.J. The Grapsoid crabs of America. Bulletin of the United States National Museum, Washington, v. 97, p. 1-461, 1918.

SUGUIO, K. Introdução a sedimentologia. New York, Edgard Blucher, 1973. 317p. 